\title{
Internal optical urethrotomy is the treatment of choice in stenosis of the bladder neck after open prostate adenectomy
}

Tomasz Borkowski, Jędrzej Michalec, Boleslaw Kuzaka, Andrzej Borkowski, Piotr Radziszewski

Department of Urology, Medical University of Warsaw, Warsaw, Poland

Videosurgery Miniinv 2019; 14 (3): 427-432

DOI: https://doi.org/10.5114/wiitm.2019.82686

\begin{abstract}
Introduction: Open adenomectomy of the prostate, although performed less frequently, is still indicated in patients with prostate adenoma $>100 \mathrm{ml}$.

Aim: This study assessed the frequency of isolated bladder neck stenosis after surgery and the effectiveness of internal optical urethrotomy as monotherapy and in combination with transurethral resection in the treatment of this complication.

Material and methods: One thousand five hundred thirty-eight Millin's operations and 381 trans-vesical adenomectomies were performed in patients with prostate adenoma. In 50 patients, the circular hemostatic suture was applied using the de la Peña technique because of bleeding after surgery. The retrospective analysis compared the incidence of isolated bladder neck stenosis depending on the type of surgery.

Results: Isolated bladder neck stenosis or narrowing of the neck combined with partial stenosis of the site after adenomectomy occurred in $0.52 \%$ (8/1539) of patients after Millin's operation and in $1.05 \%$ of patients (4/381) after trans-vesical adenomectomy. All strictures of the bladder after trans-vesical surgery occurred within 12 month after the procedure, and 25\% of stenoses after Millin's operation occurred many years after the surgery. Internal optical urethrotomy as monotherapy or in combination with scar resection resulted in recovery after one treatment in 16 out of 17 patients.

Conclusions: Internal optical urethrotomy as monotherapy or in combination with scar resection was effective in nearly all patients with bladder neck stenosis.
\end{abstract}

Key words: benign prostatic hyperplasia, adenomectomy, internal optical urethrotomy, bladder neck stenosis.

\section{Introduction}

Transurethral resection of the prostate (TURP) still remains the gold standard of treatment for lower urinary tract symptoms secondary to benign prostate obstruction in patients with prostate volume of 30-80 ml. However, the frequency of TURP, compared to other treatments, is gradually decreasing (81\% in 1999, 39\% in 2005), mainly due to effective pharmacotherapy and minimally invasive laser procedures [1].
The need for reoperation after TURP (retreatment rate) in the analysis of 20671 patients who underwent surgery was around 1-2\% per year [2]. Reinterventions (another TURP, urethrotomy, bladder neck incision) at 1, 5 and 8 years were required in $5.8 \%, 12.3 \%$ and $14.7 \%$ of cases respectively. With increasing volume of prostate adenoma radicality of resection is more difficult to achieve as more tissue has to be resected and this in turn increases the risk for reoperation. Therefore, according to the EUA Guidelines 2017, in the absence of the holmium

\footnotetext{
Address for correspondence

Tomasz Borkowski MD, Department of Urology, Medical University of Warsaw, 4 Lindleya St, 02-005 Warsaw, Poland,

phone: +48 2250217 51, e-mail: tomasz.borkowski@wum.edu.pl
} 
laser, open surgery remains the treatment of choice for large prostatic adenomas (> 80-100 ml).

Open prostatectomy (adenomectomy) is associated with high clinical and symptomatic efficiency: the improvement in LUTS by $63-86 \%, 60-87 \%$ improvement in quality of life, increase of the urinary flow up to $375 \%(16.5-20.2 \mathrm{ml} / \mathrm{s})$ and the reduction of residual volume by $86-98 \%$ [3]. Three randomized trials with a relatively small number of cases and follow-up of 78-260 weeks comparing open adenomectomy with holmium laser enucleation or photoselective vaporization of the prostate have shown that there is no need for reoperation after open procedures [4-6]. Earlier studies comparing open operations with TURP had revealed however that $2.1 \%$ to $9.5 \%$ of patients submitted to open adenomectomy required subsequent reintervention. Nevertheless, in each of these studies, the proportion of reoperation after open adenomectomy was significantly lower than the percentage of reoperation after TURP [1, 7-10].

\section{Aim}

Most studies give the total percentage of reoperation with no stratification between repeated resections or bladder neck and urethra incision. The objective of this study was to evaluate only the frequency of the isolated stenosis of the bladder neck, with or without narrowing of prostatic fossa. We tried to determine whether this complication depends uniquely on the surgical technique or other factors and to determine the role of internal urethrotomy (optical urethrotomy) in the treatment of this complication.

\section{Material and methods}

In the years 1994-2013, at the Department of Urology Medical University of Warsaw 1919 open adenomectomies were performed: 1538 retropubic with Millin's technique and 381 suprapubic through the transvesical approach. In the 1990s, adenomas of various sizes were submitted to open surgery while in recent years, only those with prostate volume exceeding $80 \mathrm{ml}$ were. In 50 patients an additional hemostatic suture had to be applied on the bladder neck according to the technique of de la Peña [11] because of severe bleeding, either at the time of primary surgery or during an urgent reoperation. This method was applied after both transvesical and Millin procedures.

\section{Step-by-step description of surgical technique}

Retropubic adenomectomy of the prostate using the Millin technique: transverse incision of the prostate capsule is performed, finger enucleation of the adenoma is carried out, 2 haemostatic sutures embracing the vesical neck and prostate capsule at the 5 and 7 o'clock positions are inserted, 2 retrigonization stitches by which the mucosa of the bladder trigone is attached to the prostatic fossa are inserted, and the prostate capsule is closed. In the postoperative course constant flow through the catheter is maintained until clean urine is obtained. The catheter is left in the bladder on average for 3 days.

Transvesical adenomectomy of the prostate: the bladder is incised longitudinally in the midline, the circular coagulation of the vesical mucosa around the internal opening of the urethra and disruption of the anterior commissure of the prostate are made. Adenoma is digitally enucleated. Several hemostatic sutures are inserted on the edges of the prostatic fossa. The incision of the bladder is closed in two layers. A three-way catheter with a balloon is kept in the bladder for 7 days on average. In the case of severe bleeding, the traction on the catheter enables for compression of the bladder neck vessels with a balloon in the bladder.

The hemostatic purse-string suture technique (de la Peña): in the case of severe bleeding during the surgery or immediately afterwards a circular suture is placed around the vesical edges of the prostatic fossa. Both ends are passed through the anterior wall of the bladder and pulled over so that the bladder neck can be tightened around the three-way catheter with the balloon positioned in the bladder. The suture is loosened on the first day after surgery and completely removed on the $2^{\text {nd }}$ or $3^{\text {rd }}$ day. After suture removal, the balloon of the catheter is filled with $5-10 \mathrm{ml}$ of the fluid and pulled down to the prostatic fossa in order to "open" the neck of the bladder. The catheter is kept in the bladder for 7 to 10 days.

Techniques for the treatment of stenosis: the stenosis is cut open with the blade of the Sachse urethrotome (Photos 1 and 2) mostly at the 12 o'clock position and occasionally at 6 and 9 o'clock positions. In the case of total obstruction, the Benique dilator is inserted through the opening in the suprapubic bladder fistula into the posterior part of the urethra. It is headed towards the direction of obstruction and 


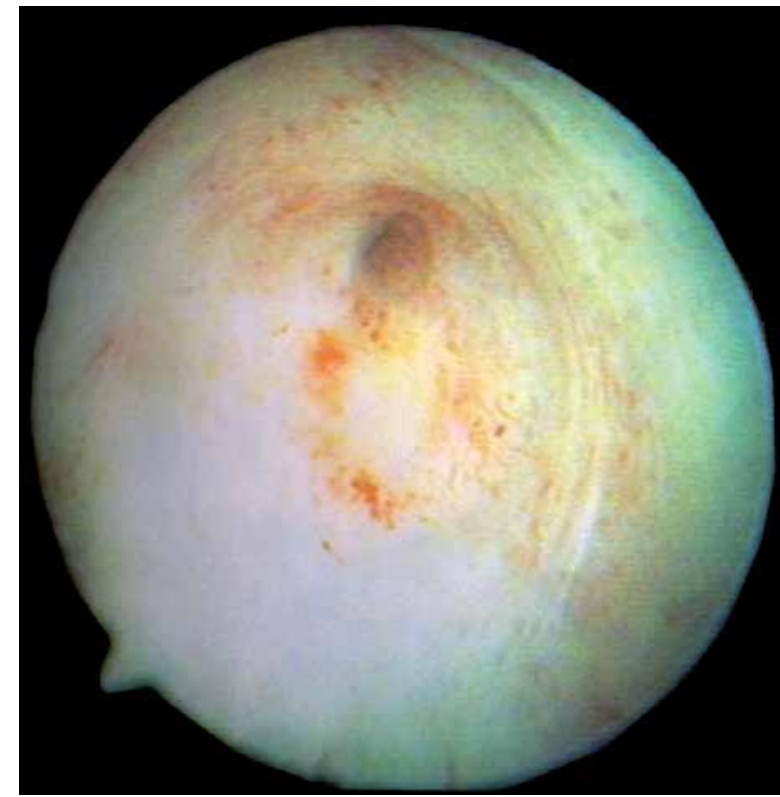

Photo 1. Stenosis of the bladder neck before incision

the scar is "blindly" transected. If the opening of the bladder neck seemed to be insufficient after incision, an electroresector is introduced, and additional deep cuts are made and the scar tissue is resected. A catheter is kept for 1-14 days after surgery.

\section{Results}

Among 1539 Millin operations carried out in the department between the years 1994-2015, $10(0.65 \%)$ patients had developed stenosis of the bladder neck. Among 381 transvesical adenomectomies, the stricture of the bladder neck occurred in $4(1.05 \%)$ patients. In addition, 5 (10\%) out of 50 patients developed narrowing of the bladder neck after the de la Peña technique, which was used in case of bleeding after both Millin's and transvesical adenomectomies. Thus, the bladder neck stenosis secondary to the use of a hemostatic suture occurred in every tenth patients and was much more frequent than after the Millin's operations and transvesical operations.

Bladder neck stenosis after the Millin's operation occurred shortly after the surgery in 5 patients, 8 and 9 months after the treatment in 2 patients, and a few years after the operation (3, 8 and 14 years) in another 3 patients. Seven patients presented the narrowing of the bladder neck within 1 year after

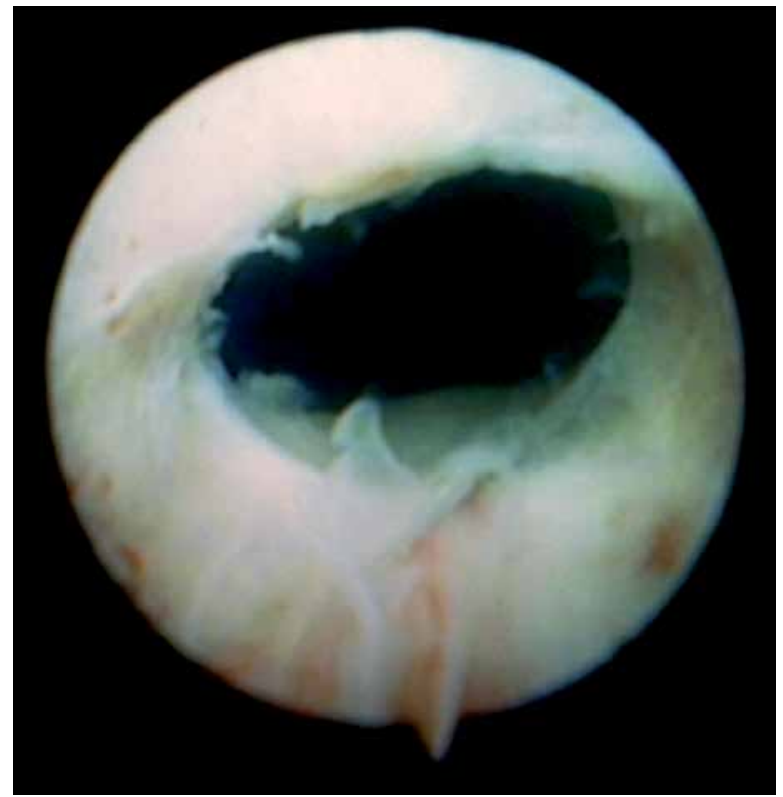

Photo 2. Stenosis is cut open with the blade of the Sachse urethrotome at the 12 o'clock position

surgery. Among them 4 had an uncomplicated enucleation and 3 with intraoperative technical complications such as: rupture of the prostate capsule or the need for "sharp" resection, because the cleavage plane of enucleation could not be found as a consequence of previous inflammation. In 3 patients who developed stenosis many years after adenomectomy, 2 had a rupture of the prostate capsule at the time of enucleation of the adenoma while in the third one the surgery was performed without any complications. In 3 patients, the narrowing was only in the bladder neck in the form of the more or less thick diaphragm with the narrow hole in the middle (Photo 3), in 6 patients bladder neck stenosis was associated with greater or lesser scarring of the prostatic urethra (Photo 4) and in 1 case the scar involved the upper part of membranous urethra, prostatic fossa and the bladder neck. In this case, extensive urethrotomy solved the problem of stenosis, but caused urinary incontinence. We noticed that in 5 out of 10 cases late complications were related to prostate capsule injury or difficult enucleation. As technically difficult cases are rare, the proportion of bladder neck stenosis seems to be disproportionately more frequent than in cases of uncomplicated surgery.

Incision of stenosis with Sachse urethrotome was sufficient in 6 patients, in the remaining 4 additional resection of scar done during the same proce- 


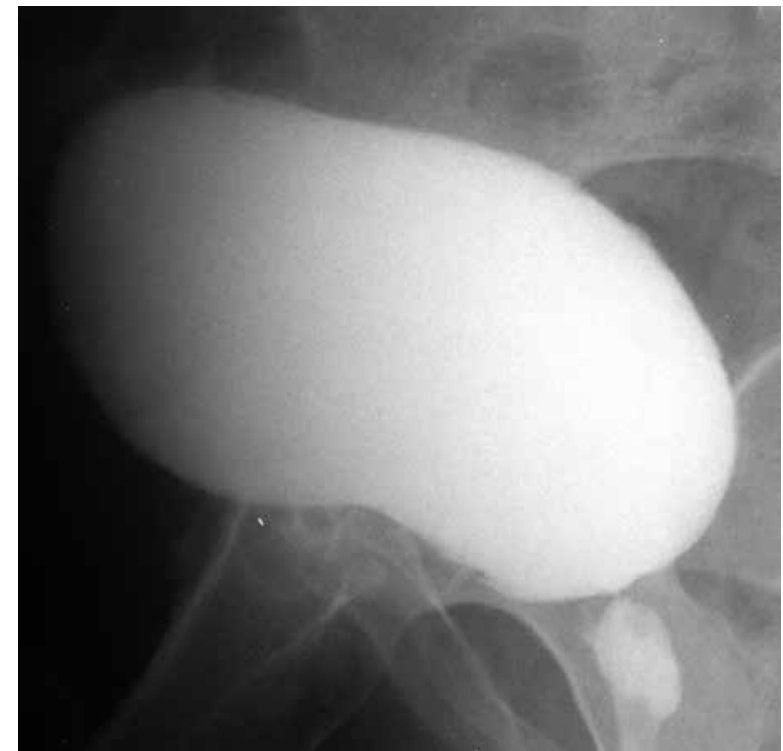

Photo 3. Urethrography: narrowing of the bladder neck only

dure was necessary. In 9 patients, a single treatment was satisfactory, in one patient urethrotomy had to be repeated 3 times with the good final result.

The narrowing of the bladder neck in transvesical surgery group occurred in 3 patients shortly after surgery and in the fourth patient within 6 months. In 3 cases enucleation of the adenoma was difficult, in another patient hemostatic sutures narrowing the prostatic fossa (different from the de la Peña technique) were used. One patient had only bladder neck narrowing, in 2 cases stenosis was present in the bladder neck and the prostatic fossa and in 1 case bladder neck, prostatic urethra and the sphincter were involved. In all patients single internal urethrotomy procedure was successful; no patient required additional resection of the scar tissue. Continence was not impaired in any case. Herein complications were probably related to technical difficulties during surgery.

In 5 patients with the use of a haemostatic suture, stenosis occurred always within first 12 months after surgery. In 3 of them only the bladder neck was narrowed, in the remaining 2 prostatic fossa was also involved. Three patients required resection of the scar in addition to internal urethrotomy. Continence was not impaired in any case.

\section{Discussion}

During last 20 years, 1919 patients underwent open adenomectomy of the prostate due to symp-

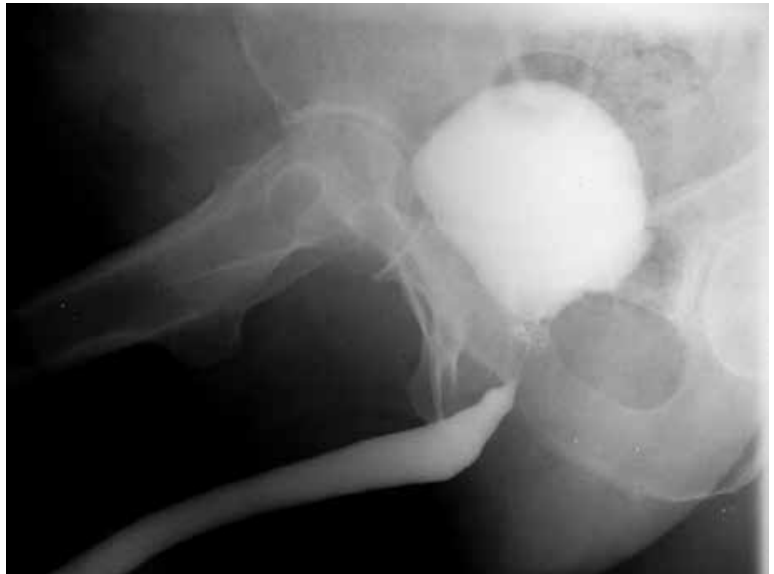

Photo 4. Urethrography: narrowing of the bladder neck associated with scarring of the prostatic urethra

tomatic benign prostatic hyperplasia (BPH). In 50 patients haemostatic suture need to be put on using the de la Peña technique, because of severe bleeding either at the time of primary surgery or few hours later in the case of urgent reoperation. This method was used both after transvesical or Millin's procedures. According to current trends, the frequency of open surgery has been steadily declining [12]. While in the years 1994-2003, 85-135 operations were performed using the Millin technique (and about 20 transvesical adenomectomies per year), in the last 5 years less than forty open adenomectomies were carried out. In 10 (0.65\%) out of 1538 of patients after the Millin's procedure, in 4 out of 381 patients (1.05\%) who underwent transvesical adenomectomy and in 5 (10\%) out of 50 patients when a hemostatic suture was applied, isolated bladder neck stenosis with or without narrowing of the prostatic fossa has been diagnosed. This analysis does not include either strictures of the urethra or regrowth or prostatic adenoma but concentrates exclusively on bladder neck strictures, which were distinguished from all other late complications of open adenomectomy.

The large retrospective study conducted by the Austrian Society of Urology assessed the cumulative percentage of re-resection of the prostate, urethrotomy or bladder neck incision secondary to 2452 adenomectomies, which were necessary in 3.8\%, 8.5\% and $9.5 \%$ after 1,5 and 8 years respectively. However, detailed percentage of the isolated bladder neck stenosis was not reported [2]. It is also worth mentioning that many of these reoperations were per- 
formed several years after surgery when regrowth of the adenoma seemed to be the main issue. In the retrospective study from Western Australia covering 19598 patients, including 18464 who underwent TURP and 1134 who underwent open adenomectomy, repeated prostatectomy was 2.3 times more common after TURP than after open surgery [9]. Another retrospective study of the Sicilian-Calabrian Society of Urology revealed a $3.6 \%$ stenosis rate of the bladder neck or urethra in a 2-year follow-up of 1804 patients who previously underwent open prostatectomy [11].

The prospective study comparing complications of 41 patients after HoLEP and 39 patients after open adenomectomy showed the cumulative percentage of stenosis of the urethra and the neck of the bladder to be in the vicinity of $5.4 \%$ after HoLEP and $5.7 \%$ after open surgery at 24 months of follow-up [4].

In the prospective study comparing 32 patients after open surgery and 42 patients after HoLEP the narrowing of the neck 5 years after surgery of large adenomas (> $100 \mathrm{ml}$ ) was distinguished from isolated strictures of the urethra only. Bladder neck stenosis occurred in $1.7 \%$ of patients after HoLEP and in $5.0 \%$ of patients after open adenomectomy [5].

The advantage of our study is that it is based on the analysis of complications after open adenomectomy in one centre only where the treatments of 1919 patients were performed always by the same technique. The full medical documentation of 20-year follow-up was available. The drawback of the study was that it was of retrospective nature, although it allowed for long-term observations. The long observation period is of utmost importance as more than $15 \%$ of stenoses occurred many years after adenomectomy. These patients would be lost from observation because the usual follow-up in prospective studies does not exceed 24 months.

Isolated bladder neck stenosis or narrowing of the bladder neck combined with partial obstruction of the prostatic fossa after open adenomectomy of the prostate is a rare complication. In our study it affected $0.65 \%$ of patients after the Millin operation and $1.05 \%$ of patients after transvesical surgery. The lower incidence of complications after the Millin operation is most likely due to easier retrigonization. The mucosal flap covering part of the circumference of the bladder neck made it less likely to create the circular scar leading to stenosis. The highest fre- quency (as high as 10\%) occurred after operations in which de la Peña hemostatic suture was applied. Even though $72 \mathrm{~h}$ after removal of the hemostatic suture the balloon of the catheter was pulled down from the bladder to the prostatic fossa in order to "open" the bladder neck, this procedure did not always prevent stricture formation. Moreover, when the hemostatic suture had to be put on, retrigonization of the prostatic fossa was not performed.

Finally, we should emphasize the high efficiency of the internal optical urethrotomy of the bladder neck as monotherapy or in combination with transurethral resection of the scar tissue. Contrary to urethral strictures, incision of the bladder neck is highly efficient as it creates a wide opening of the bladder neck and because continuous mobility during both filling up of the bladder and voiding enhances epithelisation, which in turn prevents further stenosis formation.

\section{Conclusions}

Isolated bladder neck stenosis after adenectomy or narrowing of the bladder neck combined with the stricture of the prostatic fossa are rare complications whose frequency does not exceed $1 \%$. The lowest incidence occurs after Millin's operation. It is almost twice as frequent after trans-vesical adenomectomies and much more frequent (10\%) when the purse string suture is used to achieve proper hemostasis. A single procedure of internal optical urethrotomy as monotherapy or in combination with scar resection is an effective treatment leading to recovery in almost all patients.

\section{Conflict of interest}

The authors declare no conflict of interest.

\section{References}

1. Yu X, Elliott SP, Wilt TJ, et al. Practice patterns in benign prostatic hyperplasia surgical therapy: the dramatic increase in minimally invasive technologies. J Urol 2008; 180: 241-5.

2. Madersbacher S, Lackner J, Brossner C, et al. Reoperation, myocardial infarction and mortality after transurethral and open prostatectomy: a nation-wide, long-term analysis of 23,123 cases. Eur Urol 2005; 47: 499-504.

3. Gravas S, Cornu JN, Drake MJ, et al. Management of Non-Neurogenic Male Lower Urinary Tract Symptoms (LUTS), incl. Benign Prostatic Obstruction (BPO) 2018 [Available from: http:// uroweb.org/guideline/treatment-of-non-neurogenic-maleluts/; accessed 10 May 2018. 
4. Naspro R, Suardi N, Salonia A, et al. Holmium laser enucleation of the prostate versus open prostatectomy for prostates $>70 \mathrm{~g}$ : 24-month follow-up. Eur Urol 2006; 50: 563-8.

5. Kuntz RM, Lehrich K, Ahyai SA. Holmium laser enucleation of the prostate versus open prostatectomy for prostates greater than 100 grams: 5-year follow-up results of a randomised clinical trial. Eur Urol 2008; 53: 160-6.

6. Skolarikos A, Papachristou C, Athanasiadis G, et al. Eighteen month results of a randomized prospective study comparing transurethral photoselective vaporization with transvesical open enucleation for prostatic adenomas greater than $80 \mathrm{cc}$. J Endourol 2008; 22: 2333-40.

7. Roos NP, Wennberg JE, Malenka DJ, et al. Mortality and reoperation after open and transurethral resection of the prostate for benign prostatic hyperplasia. N Engl J Med 1989; 320: 1120-4.

8. Sidney S, Quesenberry CP Jr, Sadler MC, et al. Reoperation and mortality after surgical treatment of benign prostatic hypertrophy in a large prepaid medical care program. Med Care 1992 30: $117-25$.

9. Tubaro A, De nunzio C. The current role of open surgery in $\mathrm{BPH}$. EAU-EBU Update Series 2006; 4: 191-201.

10. Semmens JB, Wisniewski ZS, Bass AJ, et al. Trends in repeat prostatectomy after surgery for benign prostate disease: application of record linkage to healthcare outcomes. BJU Int 1999 84: 972-5.

11. De La Peña A, Alcina E. Suprapubic prostatectomy: a new technique to prevent bleeding. J Urol 1962; 88: 86-90.

12. Sarma AV, Jacobson DJ, McGree ME, et al. A population based study of incidence and treatment of benign prostatic hyperplasia among residents of Olmsted County, Minnesota: 1987 to 1997. J Urol 2005; 173: 2048-53.

Received: 25.02.2018, accepted: 13.11.2018. 